\title{
Limonene-based Self-nanoemulsifying System: Formulation, Physicochemical Characterization and Stability
}

\author{
Mohammed Maher Mehanna* \\ Department of Pharmaceutical Technology, Faculty of Pharmacy, Beirut Arab University, Beirut, LEBANON.
}

\begin{abstract}
Objectives: The aim of current research was to formulate limonene selfnanoemulsified delivery system (SNEDS) by spontaneous emulsification method. Methods: The optimization was carried out through the construction of a pseudo-ternary phase diagram. Limonene-based self- nanoemulsifying system was optimized by evaluating its droplet characteristic; namely; size, polydispersity, zeta potential, in addition, its morphology was assessed through the use of transmission electron microscopy. Moreover, the formulation stability under different storage conditions for three months was examined. Results: The obtained results showed that the optimized limonene-based self-nanoemulsifying system was characterized by a small droplet size, low polydispersity index, high percentage transmittance and optimal zeta potential with uniform spherical droplets. The selected formulation with $50 \% \mathrm{w} / \mathrm{w}$ limonene, $40 \% \mathrm{w} / \mathrm{w}$ Tween 80 and $10 \% \mathrm{w} / \mathrm{w}$ propylene glycol had a droplet size of $113.3 \pm 1.18 \mathrm{~nm}$ with bluish transparent appearance and a zeta potential value of $-19.13 \pm 0.38 \mathrm{mV}$. The
\end{abstract}

developed formula was stable against $\mathrm{pH}$ change. The stored limonenebased SNEDS showed acceptable stability at $4^{\circ} \mathrm{C}$ and $0^{\circ} \mathrm{C}$ compared to $25^{\circ} \mathrm{C}$. Conclusion: The formulated self-nanoemulsifying system showed an improved aqueous dispersibility, patient acceptability and stability of limonene, representing a promising carrier for lipophilic drugs.

Key words: D-Limonene, Essential oil, Formulation, Nanoemulsion, Selfnanoemulsifying system.

\section{Correspondence}

Dr. Mohammed M Mehanna

Department of Pharmaceutical Technology, Faculty of Pharmacy, Beirut Arab University, Beirut, LEBANON.

Phone no: +961 71708661

Email:mmhanna@bau.edu.lb

DOI: 10.5530/ijpi.2020.1.12

\section{INTRODUCTION}

Phytomedicine have witnessed a tremendous progress and the world now is moving toward the use of herbal medicine or phytomedicine to strengthen the physiological systems. Essential oils extracted from plants are mixtures of volatile compounds, containing hundreds of bioactive chemical constituents mainly mono- and sesquiterpenoids and phenylpropanoids. These oils exert antifungal, antibacterial, gastroprotective, anticancer and many other therapeutically effects. ${ }^{1}$ Several studies have focused on the benefits of these phytochemicals and their effect on human health. For example, the minimum inhibitory concentration of oregano essential oil against multiple drug-resistant Escherichia coli was $0.5 \mu \mathrm{ml}^{-1}$. Thyme essential oil obtained from Thymus vulgaris L. strongly inhibited the growth of clinical strains of Staphylococcus, Enterococcus, Escherichia and Pseudomonas genus. ${ }^{2}$

D-limonene (1-methyl-4-isopropyl-cyclohexene) is a cyclic monoterpene of molecular formula $\mathrm{C}_{10} \mathrm{H}_{16}$. It is the major constituent of the essential oil extracted from the peels of citrus fruits (family Rutaceae). It has a pleasant citric fragrance and widely used for flavoring food and personal care items. It is an optically active compound that has two enantiomeric forms; $\mathrm{R}$ and $\mathrm{S}$, where the $\mathrm{R}-(+)$ is the one known as $\mathrm{D}$ limonene (Figure 1). ${ }^{3}$ A study was conducted to evaluate the difference in the bioactivity of limonene enantiomers and the results showed that (+) limonene was active against 25 different Gram positive and Gram negative bacterial species and eight different fungal species more than the (-) limonene. ${ }^{4} \mathrm{D}$-limonene is classified by the Federal regulations as safe (GRAS). ${ }^{5}$

The therapeutic effects of limonene have been studied extensively, including antioxidant, anti-inflammatory, antidiabetic, anticancer and gastroprotection. Espina et al. reported that limonene provided a bactericidal activity against Escherichia coli. ${ }^{6}$ Limonene also exhibits antifungal activity, which was evaluated by Chee et al. where the minimum inhibitory concentration (MIC) of limonene against Trichophyton rubrum was $0.5 \% \mathrm{v} / \mathrm{v}$ with a fungicidal activity. ${ }^{7}$ The anti-inflammatory effect of limonene was further explored in D'Alessio et al. study which demonstrated the ability of D-limonene to significantly reduce inflammation scores when compared to ibuprofen by decreasing tumour necrosis factor alpha (TNf- $\alpha$ ) and interleukin 6 (IL-6) concentrations. ${ }^{8}$ Plant extracts are frequently assessed for their chemotherapeutic and chemo preventive effects, especially limonene that has been studied for its chemotherapeutic effects against different types of cancer. Yu et al. showed that D-limonene inhibited the growth of the lung cancer cells and the transplanted tumor in mice, by inducing apoptosis and promoting autophagy. ${ }^{9}$ The anticancer effect of D-limonene and berberine against human gastric carcinoma cell line MGC803 was approved by Zhang et al. where D-limonene alone inhibited the growth of MGC803 cells and its combination with berberine showed remarkable synergistic anticancer effect through reactive oxygen species production, cell-cycle arrest and apoptosis induction via mitochondria-mediated intrinsic pathway. ${ }^{10}$ $\mathrm{D}$-limonene is a volatile oil, unstable in the presence of high temperature, light, air or humidity and thus it requires special preparation and formulation. Ghasemi et al. used protective encapsulation method for this bioactive compound, where it was encapsulated into whey protein and pectin at different $\mathrm{pH}$ values to form stable nanocomplexes. ${ }^{11}$ However, high energy methods apply disruptive forces and the level of energy required to produce nanoscaled droplet size is very high and thus high cost, also it has been claimed that nanoemulsion preparation using high energy method requires higher oil-to-surfactant ratio unlike the one prepared by low energy methods. ${ }^{12}$ 


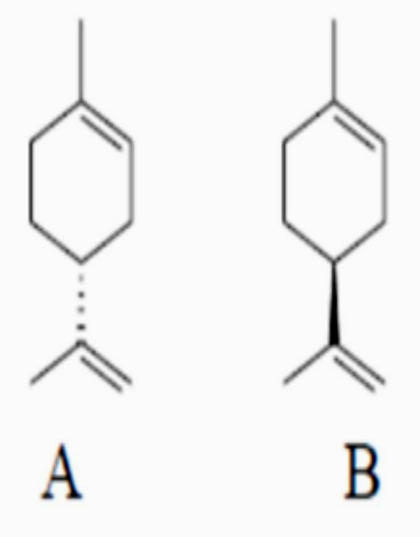

Figure 1: Structure of $\mathrm{R}-(+)$ limonene (A) and S-(-) limonene (B).

The aim of this study is to formulate limonene self-nanoemulsifying delivery system by spontaneous emulsification method. The formulation will be carried out through the construction of a pseudoternary phase diagram. Limonene-based self-nanoemulsifying system will be optimized by evaluating its droplet characteristics; namely; size, surface potential and polydispersity index. The surface morphology of the globules will be assessed through the use of transmittance electron microscopy. In addition, the formulation stability in different storage conditions for three months will be examined.

\section{MATERIALS AND METHODS}

\section{Materials}

R-(+)-Limonene, propylene glycol, polyethylene glycol 400 (PEG 400), PEG-40 hydrogenated castor oil (Cremophor ${ }^{\circ} \mathrm{RH} 40$ ) and Tween 80 were purchased from Sigma Co.(Sigma-Aldrich, Steinheim, Switzerland). PEG-8 caprylic/capric glycerides (Labrasol'), oleoyl polyoxyl-6 glycerides (Labrafil'M 1944 CS), diethylene glycol monoethyl ether (Transcutol HP') were kindly donated by Gattefosse Co. (Lyon, France). All other reagents and solvents used were of analytical grade.

\section{Preliminary screening of surfactants and co-surfactants}

The self-emulsification ability of various surfactants and the selection of the suitable surfactant were based on its emulsification power. Surfactants were screened according to method described by Date and Nagarsenker with some modifications..$^{13}$ Briefly, equal weights of each surfactant were added to limonene. The mixture was then heated at $45 \pm 2^{\circ} \mathrm{C}$ with controlled mixing (30 rpm) using magnetic stirrer till homogenization. Sample of the mixture was diluted with deionized water to $100 \mathrm{ml}$ in stoppered conical flask. The emulsification was judged according to the numbers of flask inversions required to produce a uniform emulsion. Emulsions were kept for $2 \mathrm{~h}$ and then their transmittance was measured at $638.2 \mathrm{~nm}$ (Jasco V-730 spectrophotometer) using deionized water as a blank. Then, a mixture of co-surfactant, the selected surfactant and limonene at a ratio of $1: 2: 3$ respectively were mixed at $45 \pm 2^{\circ} \mathrm{C}$ using a magnetic stirrer (30 rpm) with controlled temperature for complete homogenization. Sample of the mixture was diluted with deionized water to $100 \mathrm{ml}$ in stoppered conical flask. The self-emulsification was judged as previously described.

\section{Construction of pseudoternary phase diagram}

Constructing a pseudoternary phase diagram is a vital tool in the preparation of any lipid-based formulation that is able to self-emulsify in the body to form a thermodynamically stable nanocarrier. It will assess different phase behavior data of the formulation between different components. Limonene, the selected surfactant and co-surfactant were used to construct the ternary phase diagram using Chemix school software version 7.0 (Arne Standnes, Bergen, Norway). The concentrations of surfactant and oil varied between $30 \%$ and $70 \% \mathrm{w} / \mathrm{w}$ and that of co-surfactant ranged from $0 \%$ to $30 \% \mathrm{w} / \mathrm{w}$ according to Date and Nagarsenker. ${ }^{13}$ The mixtures were prepared by mixing surfactant/co-surfactant then the oil was added to the mixture and homogenized on a magnetic stirrer at $45 \pm 2^{\circ} \mathrm{C}$ for $15 \mathrm{~min} .{ }^{14}$ From each mixture, $50 \mathrm{mg}$ were diluted to $50 \mathrm{ml}$ with deionized water. The aqueous dispersions that are transparent to slightly bluish with a particle size of $200 \mathrm{~nm}$ or less were considered in the accepted region of the phase diagram. ${ }^{14}$ The droplet size determination was carried out using Zeta sizer 2000 (Malvern Instruments, UK).

\section{Preparation of limonene-based self-nanoemulsifying delivery system}

The selected formula was prepared by mixing surfactant and co-surfactant and then adding the oil drop wise. Heating the mixture for $15 \mathrm{~min}$ on a magnetic stirrer at $30 \mathrm{rpm}$ with controlled temperature at $45 \pm 2^{\circ} \mathrm{C}$ was carried out. The preparations were stored in sealed glass vials at room temperature for further investigations.

\section{Study of SNEDS characteristics}

Color, transparency, phase separation and homogeneity of the preparation at room temperature were observed for any changes that might occur. All tests were performed in set of three. Turbidity measurement was performed as the following; prepared formulation was diluted into $100 \mathrm{ml}$ with deionized water. The percentage transmission was measured spectrophotometrically at $638.2 \mathrm{~nm}$. In addition, the self-emulsification time of the prepared SNEDS was determined by adding $1 \mathrm{ml}$ of the preparation to $300 \mathrm{ml}$ deionized water at $37 \pm 0.5^{\circ} \mathrm{C}$ in a beaker stirred at $30 \mathrm{rpm}$. The time required for this concentrate to form a homogeneous clear bluish mixture is recorded as the emulsification time. The selected formulation was visually assessed and classified accordingly to the following criteria; rapidly forming emulsion having a clear or bluish appearance (grade A), rapidly forming, slightly less clear emulsion, having a bluish white appearance (grade B), fine milky emulsion formed within two minutes (grade C), dull, greyish white emulsion having slightly oily appearance that is slow to emulsify longer than two minutes (grade D), or poor minimal emulsification with large oil globules present on the surface (grade E) ${ }^{15}$ In order to determine the formulation the cloud point, the prepared formulation was diluted with deionized water in ratio of 1:100 then placed in a water-bath (FALC, WB-MF24, Treviglio (BG) - Italy) with gradual increase in the temperature at a rate of $1^{\circ} \mathrm{C} /$ min. At the cloud point, the drop in sample percentage transmittance was measured spectrophotometrically at $638.2 \mathrm{~nm} .{ }^{14}$

Moreover, the surface morphology and globule size of the optimized limonene-based SNEDS were observed by TEM (Jeol, JEM-100 CX electron microscope). The sample was diluted with water (1:1000) then a drop was placed on the copper grid. Excess sample was removed with filter paper. The grid was dried at room temperature and then observed using TEM. Zeta potential, droplet size and polydispersity index (PDI) were measured at $25 \pm 2^{\circ} \mathrm{C}$ and at $90^{\circ}$ scattering angle by Zeta sizer 2000 (Malvern Instruments, UK) after the dilution of the formulation to 100 $\mathrm{ml}$ with deionized water.

\section{Evaluation of the self-nanoemulsifying delivery system}

The robustness of the preparation to dilution was performed through diluting the formulation 50,100 and 1000 times with different media, namely; deionized water, $0.1 \mathrm{~N} \mathrm{HCl}(\mathrm{pH} 1.2)$ and phosphate buffer 
( $\mathrm{pH}$ 7.4). The diluted preparations were stored for $12 \mathrm{~h}$ at $25 \pm 2^{\circ} \mathrm{C}$ and observed for any signs of phase separation or precipitation. ${ }^{16}$ In addition the Thermodynamic stability studies were performed as following testing the formulation stability. The prepared formulation after dilution to $100 \mathrm{ml}$ with deionized water was exposed to centrifugation at 5000 rpm for 30 min to observe any changes in its homogeneity. In addition, stability was challenged through six freeze-thawing cycles between refrigerator temperature $\left(4 \pm 0.5^{\circ} \mathrm{C}\right)$ and $\left(25 \pm 0.5^{\circ} \mathrm{C}\right)$ with storage at each temperature for $48 \mathrm{hr}$.

\section{Stability upon storage}

The stability of the formulation was tested through its storage at room temperature at $25 \pm 2^{\circ} \mathrm{C}$, at $4 \pm 2^{\circ} \mathrm{C}$ and at $0 \pm 2^{\circ} \mathrm{C}$ for three months and monitored for any signs of separation or precipitation. Limonene based SNEDS samples were assessed every four weeks and analyzed for clarity, particle size, PDI and zeta potential.

\section{Statistical analysis}

Results were expressed as mean \pm standard deviation $(n=3)$ and compared for statistical significance using $t$-test or one-way-analysis of variance (ANOVA) test. $p$-value $<0.05$ was considered to be significant using GraphPad Prism version 8.0.2 (GraphPad Software, San Diego, CA).

\section{RESULTS}

\section{Selection of surfactants and co-surfactants}

The choice of surfactant and co-surfactants was based on the optical clarity of the nano-dispersion, reflected by high transmittance, where the intensity of the scattered light passing through dispersion increases with the presence of optical homogeneities in the medium. The percentage transmittance of the limonene preparations using different vehicles are represented in Figure 2, revealed that the preparation with Tween 80 as surfactant and propylene glycol as co-surfactant showed highest percentage transmittance compared to other vehicles, $76.7 \pm 0.135 \%$ and $50.57 \pm 0.16 \%$ respectively with $p$-value $<0.05$ (Figure 2 ).

\section{Pseudoternary phase diagram of limonene-based SNEDS}

After selecting the optimum components to formulate limonene SNEDS, the pseudoternary phase diagram was constructed. Table 1 shows the different compositions of phase diagram mixtures meanwhile Figure 3 illustrates the pseudoternary phase diagram of limonene SNEDS. In the current investigation, although at a ratio of 1:1 (oil: surfactant) the nanoemulsifying ability was present, when the surfactant/ co-surfactant mixture was at a ratio of $4: 1$, the nanoemulsion region was wider. Formulation A10 with 50\% w/w limonene, $40 \%$ w/w Tween 80 and $10 \% \mathrm{w} / \mathrm{w}$ propylene glycol had the smallest droplet size $(113.3 \pm 1.18 \mathrm{~nm})$ with bluish transparent appearance was chosen for the further studies.

\section{Physiochemical characteristics and evaluation of limonene-based SNEDS}

The physiochemical characteristics of the optimized limonene-based SNEDS are presented in Table 2. The percent transmittance after dilution with deionized water was $92.56 \%$ with a clear bluish to slightly white solution. With a simple agitation at $37 \pm 0.5^{\circ} \mathrm{C}$, the self-emulsification time of limonene-based SNEDS was $37 \mathrm{sec}$ as shown in Table 2. Visual observations showed that the formulation was found to be grade A. Formulation was diluted with $0.1 \mathrm{~N} \mathrm{HCl}$, phosphate buffer $\mathrm{pH} 7.4$ and deionized water to 50-, 100- and 1000 folds and showed no signs of precipitation, separation or cloudiness for $12 \mathrm{~h}$ except for one with deionized water that

Table 1: Composition of SNEDS formulations constructing phase diagram.

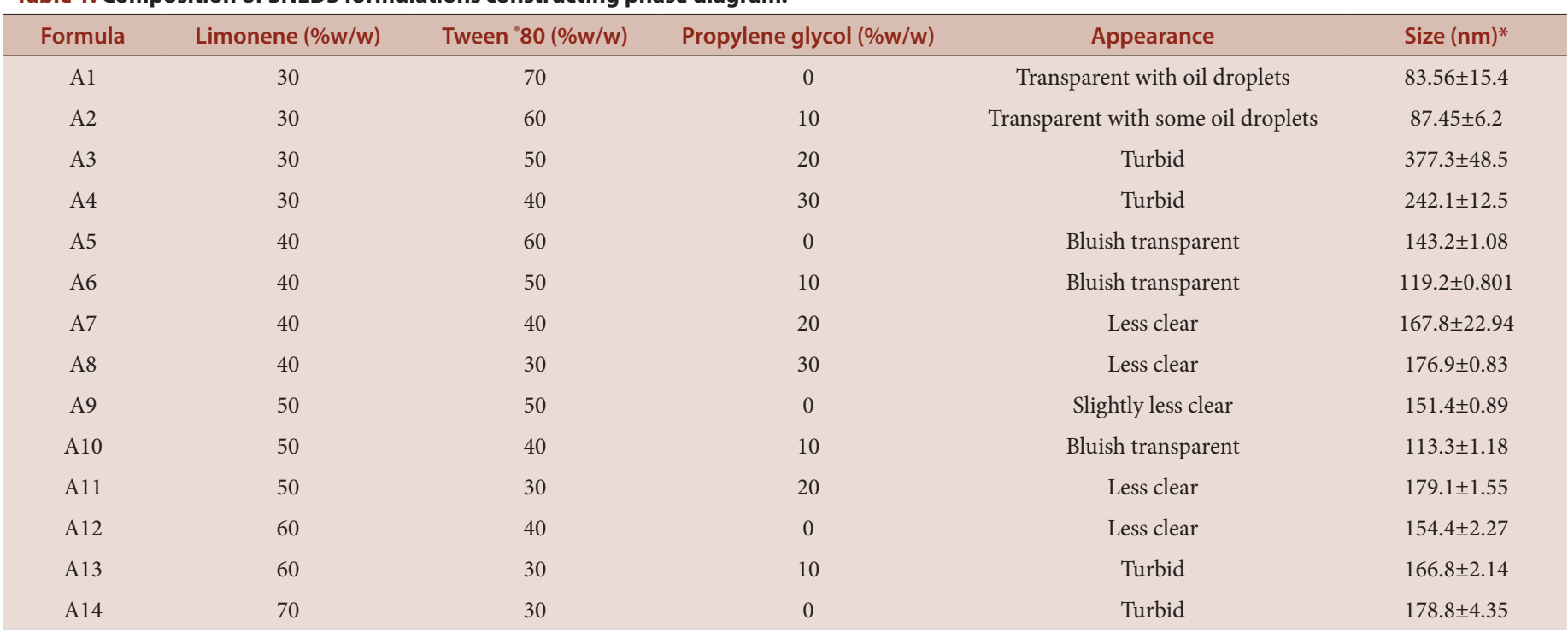

${ }^{*}$ Results are expressed as mean $\pm \mathrm{SD}(n=3)$

Table 2: Physiochemical characteristics of limonene-based SNEDS.*

\begin{tabular}{ccccccc}
\hline Formula & $\begin{array}{c}\text { Percentage } \\
\text { Transmittance }\end{array}$ & Droplet size $(\mathrm{nm})$ & PDI index & $\begin{array}{c}\text { Zeta potential } \\
(\mathrm{mV})\end{array}$ & $\begin{array}{c}\text { Self-emulsification time } \\
(\mathrm{sec})\end{array}$ & $\begin{array}{c}\text { Cloud point } \\
\left({ }^{\circ} \mathrm{C}\right)\end{array}$ \\
\hline A10 & $92.57 \pm 0.98$ & $113.30 \pm 1.18$ & $0.211 \pm 0.007$ & $-19.13 \pm 0.38$ & $37 \pm 2.52 \mathrm{Grade}(\mathrm{A})$ & $72 \pm 1.73$ \\
\hline
\end{tabular}

${ }^{*}$ Results are expressed as mean \pm SD $(n=3)$ 

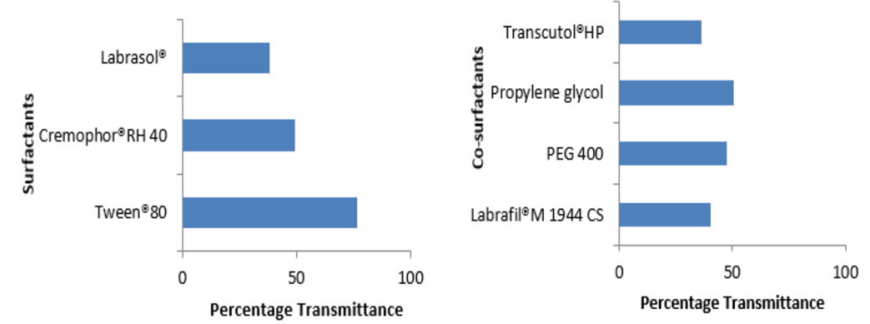

Figure 2: Preliminary screening of different surfactants and co-surfactants based on their percentage transmittance.

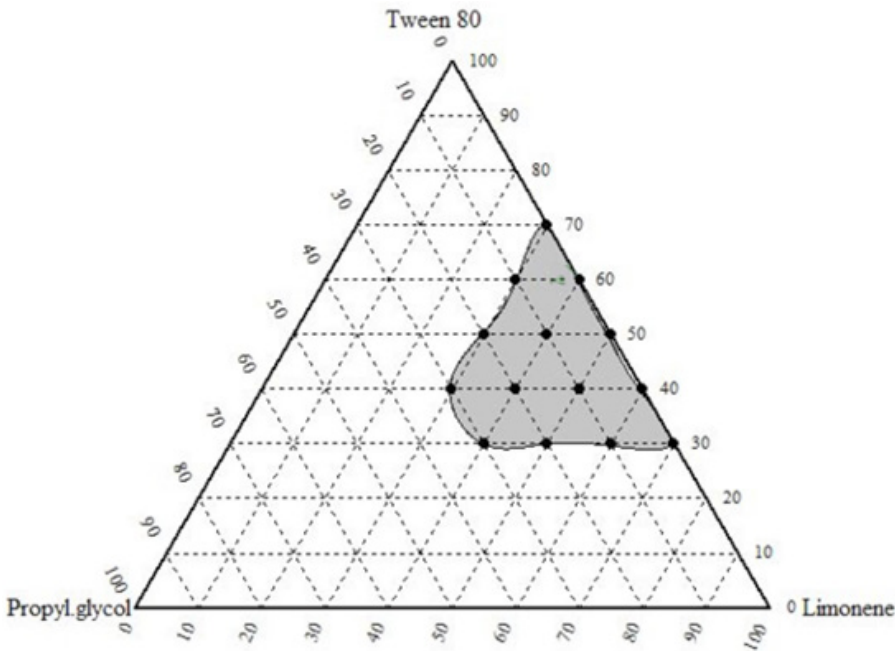

Figure 3: Pseudoternary phase diagram of limonene-based SNEDS. The shaded region indicates the nanoemulsion region.

showed slight creaming which was reversible with simple flask inversion (Figure 4).

The formulation showed high stability and no signs of precipitation or phase separation under drastic stressful conditions, specifically, centrifugation at $5000 \mathrm{rpm}$ for $30 \mathrm{~min}$, six cycles of freeze-thawing between temperature $4^{\circ} \mathrm{C}$ and $25^{\circ} \mathrm{C}$. The optimized limonene-based SNEDS was stable despite the increase in temperature up to $72^{\circ} \mathrm{C}$ and its percentage transmittance didn't change and remained above $90 \%$. The morphology of the limonene-based SNEDS was observed using TEM. As the microphotograph represented in Figure 5, globules after dilution showed spherical shape with diameter less than $200 \mathrm{~nm}$ and appeared as bright particles with dark surrounding. In the current study, limonene-based SNEDS had small droplet size less than 200nm as shown in Table 2 and Figure 5 . The polydispersity index for the formulation was 0.211 with a unimodal distribution and with a zeta potential value of $-19.13 \pm 0.38$ $\mathrm{mV}$ Figure 6.

\section{Storage stability testing}

Limonene-based SNEDS didn't show any change in its visual appearance upon storage, there was no precipitation or flocculation or phase separation. Globule size, PDI, zeta potential and percentage transmittance remained stable at both $4 \pm 2^{\circ} \mathrm{C}$ and $0 \pm 2^{\circ} \mathrm{C}$ within three months storage period; however storage at room temperature $\left(25 \pm 2^{\circ} \mathrm{C}\right)$ didn't favor the stability of the formulated limonene-based SNEDS as size and PDI increased from $113.6 \pm 1.18 \mathrm{~nm}$ and $0.211 \pm 0.007$ to $167.73 \pm 29.44 \mathrm{~nm}$ and $0.88 \pm 0.01$, respectively as illustrated in Figures 7 and 8 .
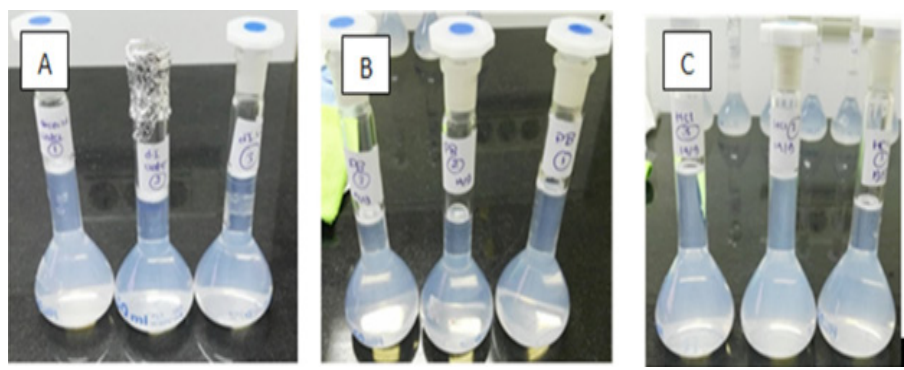

Figure 4: Limonene based SNEDS after 24hr dilution with (A) deionized water, (B) phosphate buffer, pH7.4 and (C) 0.1 N HCl, pH 1.2.

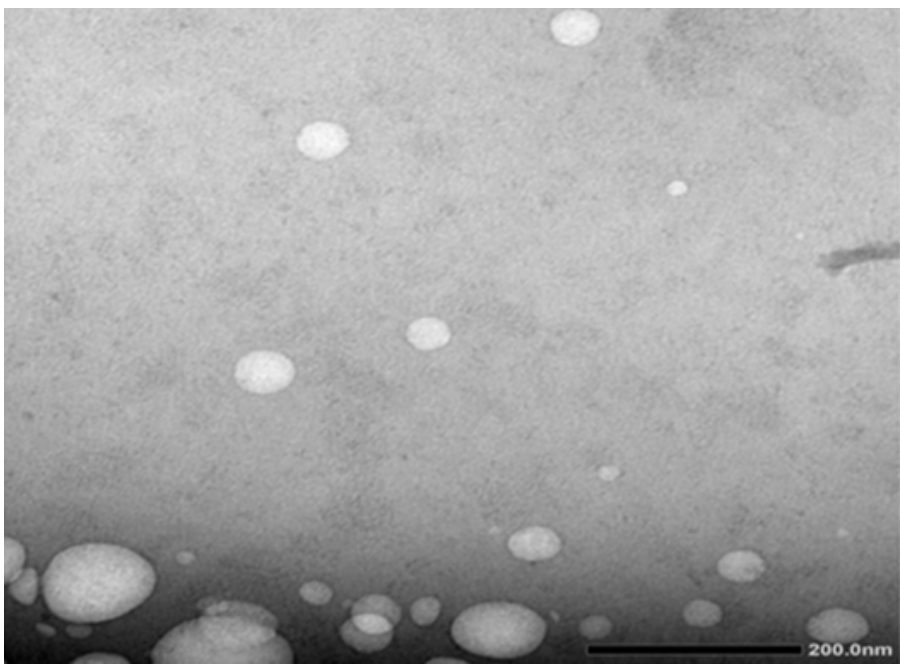

Figure 5: Photoelectromicroscopic image limonene-based SNEDS using transmission electron microscopy.
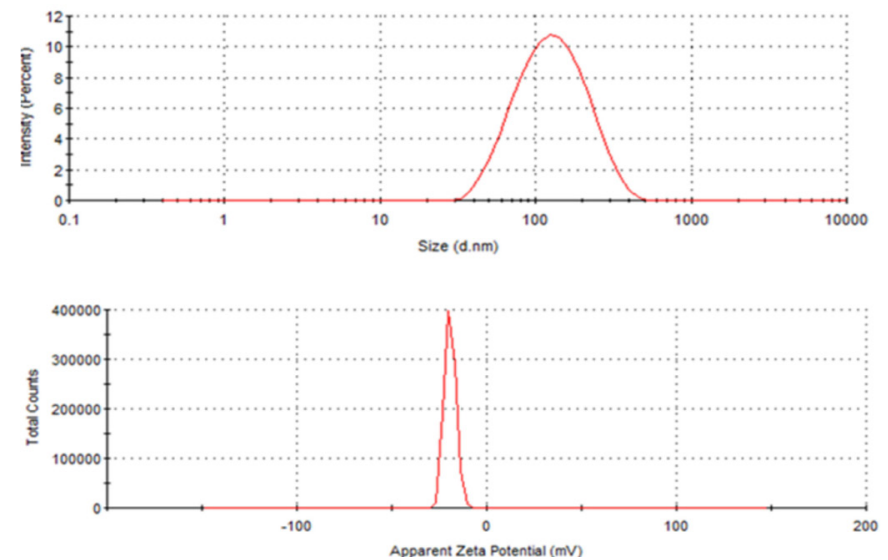

Figure 6: Droplet size distribution and zeta potential of optimized limonenebased SNEDS (formula A10).

\section{DISCUSSION}

Self-nanoemulsifying drug delivery system is widely used in the pharmaceutical field. This system safely and effectively increases drug bioavailability. ${ }^{17}$ Natural components have received great interest due to their low toxicity, pharmacological activity and economical availability. ${ }^{18}$ In the current study, three non-ionic surfactants were of HLB in the range of 12-16 and such values resulted in the formation of a stable $\mathrm{o} / \mathrm{w}$ nanoemulsion. Aqueous dispersions with high transmittance were 

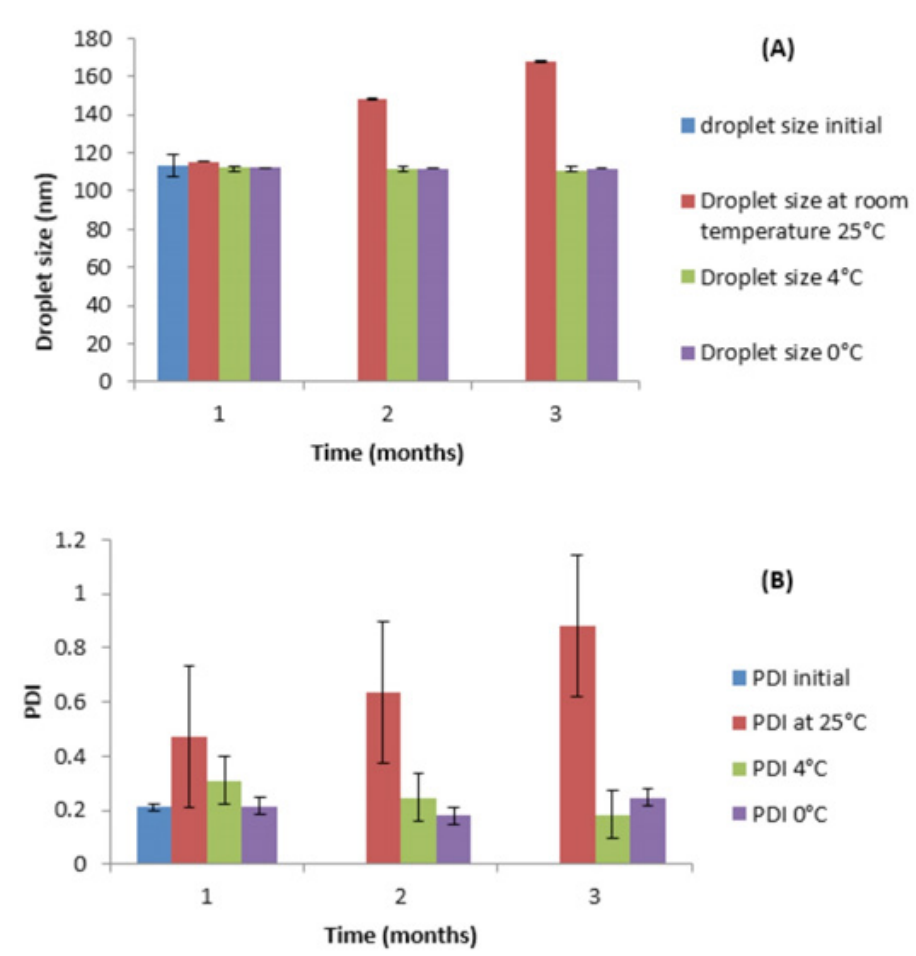

Figure 7: Droplet size (A) and PDI (B) of limonene-based SNEDS for three months storage period at different temperatures.
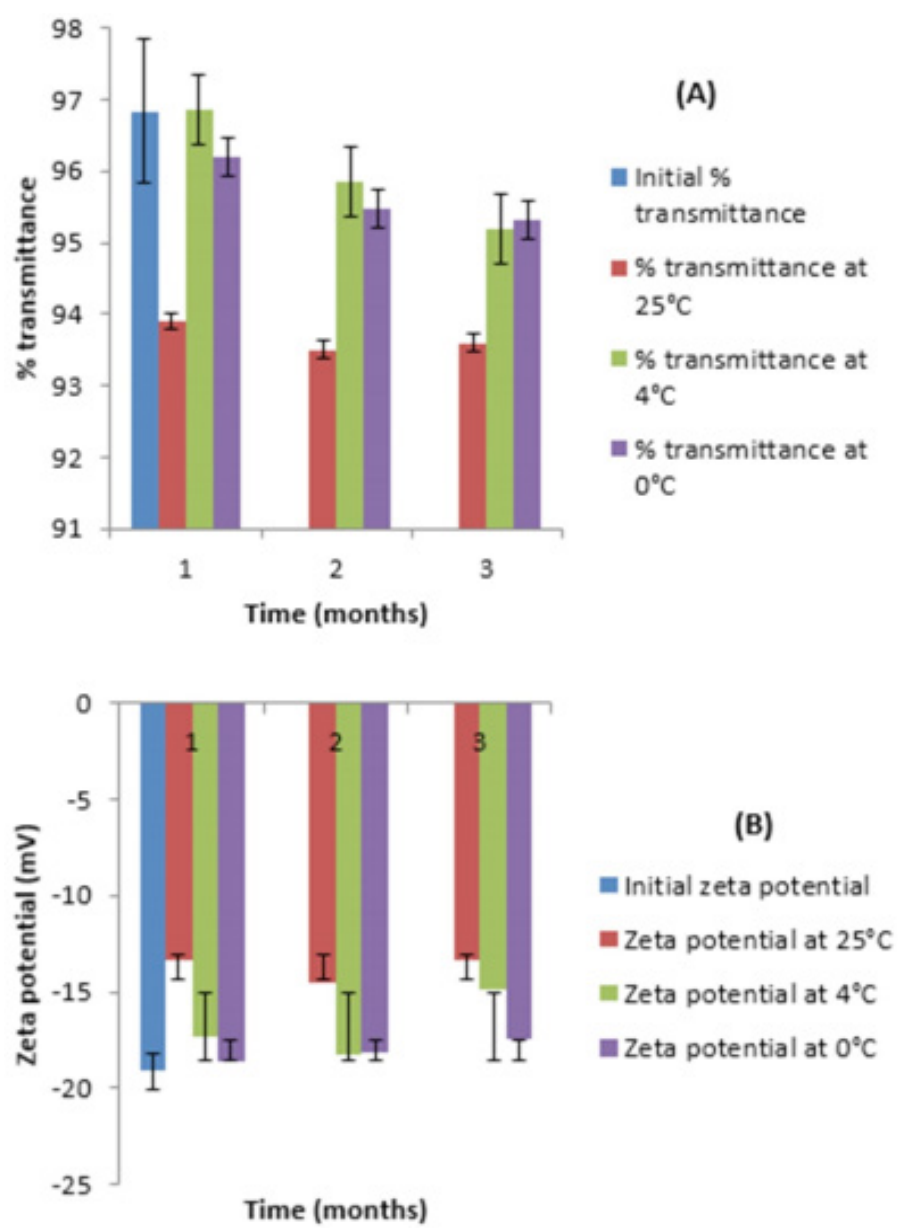

Figure 8: Percentage transmittance (A) and zeta potential (B) of limonenebased SNEDS for three months storage period at different temperatures. classified as optically clear and oil droplets were assumed to be in a nanodispersion state. ${ }^{19}$ Tween 80 exhibited the highest emulsification power compared ( $p$-value $<0.0001$ ) thus it was selected for the formulation of the SNEDS.

Addition of co-surfactants is believed to enhance the emulsification ability of the surfactant, reduces its concentration, as well as providing more stability. ${ }^{20}$ Besides, co-surfactant decreases the bending stress of the interface and enhances its flexibility and allows its occurrence at different curvatures. $^{21}$ The highest emulsification ability was reported for propylene glycol followed by PEG400. Although the addition of co-surfactant didn't increase the percent transmittance of the dispersions, yet the incorporation of short-to medium chain co-surfactants enhanced nanoemulsion region, stability and obtained nanoemulsion at low surfactant concentration. ${ }^{22}$ On the pseudoternary diagram, the shaded region at ratio 4:1 was the nanoemulsion region indicating a good self-nanoemulsifying capability. This could be due to the incorporation of co-surfactant and its effect on the penetration of the oil phase into the hydrophobic region of the surfactant which decreased the interfacial tension and increased fluidity of the interface and the system entropy as previously reported by Akhtar et al. ${ }^{23}$ Thus, formula A10 with $50 \% \mathrm{w} / \mathrm{w}$ limonene, $40 \% \mathrm{w} / \mathrm{w}$ Tween 80 and $10 \% \mathrm{w} / \mathrm{w}$ propylene glycol was selected.

After dilution, the formulation showed a clear bluish to transparent solution. This result was in accordance with that reported by Gaikwad. ${ }^{24}$ The purpose of the determination of self-emulsification time is to study the efficiency of nanoemulsion formation. ${ }^{16}$ With a simple agitation at $37 \pm 0.5^{\circ} \mathrm{C}$, the self-emulsification time of limonene-based SNEDS was 37 sec and the nanoemulsion was grade (A). The SNEDS was subjected to several folds of dilutions with different media, aiming at mimicking the in-vivo conditions that the formulation might encounter. ${ }^{16}$ The tested dilutions showed no signs of precipitation, separation or cloudiness for $24 \mathrm{~h}$ except for one with deionized water that showed slight creaming which was reversible with simple flask inversion. This creaming doesn't strike a problem since nanoemulsions with their small droplet size are subjected to Brownian motion rather than gravitational forces. An analogous phenomenon was observed by Klang et al. ${ }^{25}$ This stability against $\mathrm{pH}$ change is mainly attributed to the presence of non-ionic surfactants. ${ }^{26}$ Cloud point temperature study should always be tested at temperature above $37^{\circ} \mathrm{C}$ corresponding to the body temperature. The turbidity can be explained by the dehydration of the polyethylene oxide group in a nonionic surfactant causing phase separation and a drop in the percentage transmittance. Limonene-based SNEDS was stable up to $72^{\circ} \mathrm{C}$.

Transmission electron microscopy illustrated the spherical shape of the nanodroplets with diameter less than $200 \mathrm{~nm}$ indicated well packed and stable interfacial film and thus lower surface tension and rapid absorption of drug. Similar results were reported by Kazi et al. where droplet size was found to be around $150 \mathrm{~nm}$ having spherical shape. ${ }^{27}$

Another crucial parameter is polydispersity index (PDI), which reflected positively on formulation physical stability. Our results were less than 0.5 which indicates uniformity of droplet size distribution of limonene within the formulation and affirms its homogeneity. ${ }^{28}$ Zeta potential reflects the degree of repulsion between adjacent, similarly charged particles, high zeta potential assures colloidal stability. The formulation had zeta potential value of $-19.13 \pm 0.38 \mathrm{mV}$. The obtained results are in agreement with Dou et al. who prepared stable brucine SNEDS formulation with a zeta potential value of $-15.76 \pm 0.42 \mathrm{mV} .^{29}$

The main advantage for using SNEDS is its stability, where the small size of the droplets act as Brownian particles and don't interact with each other, thus providing stability for months. Limonene-based SNEDS didn't change in visual appearance upon storage at different temperatures. However, it didn't favor the storage at room temperature due to the movement of the dispersed droplet through the continuous phase 
increasing the opportunity for droplet collisions. This change in the physical characteristics is due to temperature. It also affects film compressibility by changing the solubility of the surfactants in the bulk phase. These results are in agreement with those of Eid et al. where the olive oil SNEDS stored at 4,25 and $40^{\circ} \mathrm{C}$ for six months, showed good stability at $4^{\circ} \mathrm{C}$ compared to $25^{\circ} \mathrm{C}$ and $40^{\circ} \mathrm{C} .{ }^{30}$

\section{CONCLUSION}

In the current investigation, limonene-based SNEDS was developed using low energy emulsification method. The optimized formulation was characterized by the smallest droplet size $(113.3 \mathrm{~nm})$, with a unimodel size distribution indicated by the lowest PDI (0.211), highest percentage transmittance $(92.57 \%)$ and optimal zeta potential $(-19.13 \mathrm{mV})$. The SNEDS preparation was robust to different media and volumes of dilutions. The preparation was stable during the three month of storage at temperature 0 and $4^{\circ} \mathrm{C}$, without showing any signs of phase separation, precipitation or flocculation. The data accumulated from this chapter suggested that self-nanoemulsifying drug delivery systems are potential tool for liquid lipophilic components such as essential oils. A liquid formulation such as SNEDDS is probably more suitable to be used for essential oil to enhance its stability and patient acceptability.

\section{ACKNOWLEDGEMENT}

The author is grateful for Gatefosse Corp. company for oils samples.

\section{CONFLICT OF INTEREST}

The author reports no conflict of interest.

\section{ABBREVIATIONS}

GRAS: Generally recognized as safe; HCl: Hydrochloric acid; HLB: Hydrophilic lipophilic balance; IL-6: Interleukin 6; MIC: Minimum inhibitory concentration; O/W: Oil in water; $\% \mathbf{w} / \mathbf{w}$ : Percentage weight per weight; PEG: Polyethylene glycol; PDI: Polydispersity index; $\boldsymbol{p}$-value: Probability value; rpm: Round per minute; SNEDS: Self-nanoemulsifying delivery system; SNEDDS: Self-nanoemulsifying drug delivery system; TEM: Transmission electron microscopy; TNF- $\alpha$ : Tumor necrosis factor alpha.

\section{REFERENCES}

1. Gandhi GR, Vasconcelos ABS, Haran GH, et al. Essential oils and its bioactive compounds modulating cytokines: A systematic review on anti-asthmatic and immunomodulatory properties. Phytomedicine. 2019;152854.

2. Sienkiewicz $M$, Łysakowska $M$, Denys $P$, Kowalczyk $E$. The antimicrobial activity of thyme essential oil against multidrug resistant clinical bacterial strains. Microb Drug Resist. 2012;18(2):137-48

3. Paul E, Viljoen A. Limonene - a review: Biosynthetic, ecological and pharmacological relevance. Nat Prod Commun. 2008;3(7):1193-202.

4. Lis-Balchin M, Ochocka RJ, Deans S, Asztemborska M, Hart S. Bioactivity of the enantiomers of limonene. Med Sci Res. 1996;24309-310.

5. Sun J. D-Limonene: Safety and clinical applications. Altern Med Rev. 2007;12(3):259-64

6. Espina L, Gelaw TK, DeLamo-Castellví S, Pagán R, García-Gonzalo D. Mechanism of bacterial inactivation by (+)-Limonene and its potential use in food preservation combined processes. PLoS One. 2013;8(2):1-10.

7. Chee HY, Kim H, Lee MH. In vitro antifungal activity of limonene against Tricho- phyton rubrum. Mycobiology. 2009;37(3):243-6.

8. D'Alessio P, Bisson J, Schulzke J, Ursini M, Béné M, Ostan R. Oral administration of d-Limonene controls inflammation in rat colitis and displays anti-inflammatory properties as diet supplementation in humans. Life Sci. 2013;92(2426):1151-6.

9. Yu X, Wang $Y, L v W$, et al. D-limonene exhibits antitumor activity by inducing autophagy and apoptosis in lung cancer. Onco Targets Ther. 2018;11:1833-47.

10. Zhang X, Liu D, Tang G, Zhang H, Wang L. Synergistic inhibitory effect of berberine and d-limonene on human gastric carcinoma cell line MGC803. J Med Food. 2014;17(9):955-62.

11. Ghasemi S, Assadpour E, Khomeiri M, Mahdi SJ. Nanoencapsulation of d-limonene within nanocarriers produced by pectin-whey protein complexes. Food Hydrocoll. 2018;77:152-62.

12. Solans C, Solé I. Nano-emulsions: Formation by low-energy methods. Curr Opin Colloid Interface Sci. 2012;17(5):246-54.

13. Date AA, Nagarsenker MS. Design and evaluation of self-nanoemulsifying drug delivery systems(SNEDDS) for cefpodoxime proxetil. Int J Pharm. 2007;329:166-72.

14. Elnaggar YSR, El-Massik MA, Abdallah OY. Self-nanoemulsifying drug delivery systems of tamoxifen citrate: Design and optimization. Int J Pharm. 2009;380(1-2):133-41

15. Singh S, Pathak K, Bali V. Product development studies on surface-adsorbed nanoemulsion of olmesartan medoxomil as a capsular dosage form. Am Assoc Pharm Sci. 2012;13(4):1212-21.

16. Yadav PS, Yadav E, Verma A, Amin S. Development, characterization and pharmacodynamic evaluation of hydrochlorothiazide loaded self-nanoemulsifying drug delivery systems. Sci World J. 2014;2014:1-10.

17. Khan A, Ansari S, Sharma R, Ali J, Kotta S. Potentials and challenges in selfnanoemulsifying drug delivery systems. Expert Opin. 2012;9(10):1305-17.

18. Chouhan S, Sharma K, Guleria S. Antimicrobial activity of some essential oils — present status and future perspectives. MDPI:Medicines. 2017;4(58):1-21

19. Pol A, Hegde D, Patel PA. Peppermint oil based drug delivery system of aceclofenac with improved anti-inflammatory activity and reduced ulcerogenecity. Int J Pharma Biosci Technol. 2013;1(2):89-101.

20. Nasr A, Gardouh AR, Ghonaim HM, Abdelghany E. Effect of oils, surfactants and cosurfactants on phase behavior and physiochemical properties of self-nanoemulsifying drug delivery system (SNEDDS) for Irbesartan and Olmesartan. Int J Appl Pharm. 2016;8(1):13-24.

21. Priya $S$, Koland M, Suchetha KN. Nanoemulsion components screening of quetiapine fumarate: Effect of surfactant and co surfactant. Asian J Pharm Clin Res. 2015;8(6):136-40.

22. Azeem A, Ahmad F, lqbal Z, et al. Nanoemulsion components screening and selection: A technical note. Am Assoc Pharm Sci. 2009;10(1):69-76.

23. Akhtar J, Hussain HS, Fareed S, Aqil M. Nanomulsion as a carrier for efficient delivery of metformin. Curr Drug Deliv. 2014;11(2):243-52.

24. Gaikwad NM, Shaikh KS, Chaudhari PD. Development and evaluation of a system for colonic delivery of budesonide. Indian J Pharm Educ Res. 2017;51(4):551-61.

25. Klang V, Matsko NB, Valenta C, Hofer F. Electron microscopy of nanoemulsions: An essential tool for characterisation and stability assessment. Micron. 2012;43(2-3):85-103.

26. Ozturka B, Ozilgen M, McClements DJ, Arginc S. Formation and stabilization of nanoemulsion-based vitamin $\mathrm{E}$ delivery systems using natural surfactants: Quillaja saponin and lecithin. J Food Eng. 2014;142:57-63.

27. Kazi M, Al-swairi M, Ahmad A, Raish M. Evaluation of self-nanoemulsifying drug delivery systems ( SNEDDS ) for poorly water-soluble talinolol: Preparation, in vitro and in vivo assessment. Front Pharmacolgy. 2019;101-13.

28. Mahmoud $\mathrm{H}$, Al-Suwayeh S, Elkadi S. Design and optimization of self nanoemulsifying drug delivery systems of simvastatin aiming dissolution enhancement. African J Pharm Pharmacol. 2013;7(22):1482-500.

29. Dou $Y$, Wang T, Huang $Y$, et al. Self-nanoemulsifying drug delivery system of Bruceine D: a new approach for anti-ulceratice colitis. Int J Nanomedicine. 2018;135887-5907.

30. Eid AMM, EIMarzugi NA, El-Enshasy HA. Preparation and evaluation of olive oil nanoemulsion using sucrose monoester. Int J Pharm Pharm Sci. 2013;5(3):43440. 